\title{
Takayasu-Arteriitis des Kindesalters nach allogener Stammzelltransplantation bei Hyper-IgE-Syndrom mit Nachweis einer NOD2-Mutation
}

\author{
C. Reiser ${ }^{1}$; A. Kurringer ${ }^{1}$; A. Lawitschka²; A. Schuster³; J. B.Kuemmerle-Deschner"; C. Huemer ${ }^{1}$ \\ ${ }^{1}$ Landeskrankenhaus Bregenz, Abteilung für Kinder- und Jugendheilkunde, Bregenz, Österreich; ${ }^{2}$ St. Anna Kinderspital, Medizinische Universität Wien, Wien, Österreich; ${ }^{3}$ Landes- \\ krankenhaus Bregenz, Abteilung für Radiologie, Bregenz, Österreich; ${ }^{4}$ Division of Rheumatology, Department of Pediatrics, University hospital Tuebingen und autoinflammation re- \\ ference center Tuebingen, Tuebingen, Deutschland
}

Die Takayasu-Arteriitis des Kindesalters (c-TA) als seltene Entität der Großgefäßvaskulitis ist sowohl in der initialen Diagnostik als auch im Krankheitsmanagement immer wieder herausfordernd, entsprechend hoch ist die Morbidität und Mortalität. Als eine möglicherweise erfolgversprechende Therapieoption bei therapieresistenten Vaskulitiden wird die Stammzelltransplantation in der Literatur diskutiert. In dem hier präsentierten Fall eines zehnjährigen Jungen, bei dem eine allogene hämatopoetische Stammzelltransplantation aufgrund eines schwersten Hyper-IgESyndroms durchgeführt worden war, wurde im Verlauf nach Transplantation die Diagnose einer Takayasu-Arteriitis gestellt. Das Management der immunsuppressiven Therapie gestaltete sich schwierig, inzwischen zeigt sich aber unter einer intensivierten Therapie mit Infliximab kein weiterer Progress der Erkrankung. Im Rahmen der weiteren ätiologischen Abklärung fand sich eine NOD2/CARD15 Genmutation auf Exon 4 (S506PfsX11), deren klinische Relevanz aktuell noch unklar ist. Anhand dieses Falls illustrieren wir die Schwierigkeiten des Monitorings der Krankheitsaktivität wegen mangelnder spezifischer Biomarker, den Stellenwert der radiologischen Diagnostik, die häufigsten Komplikationen sowie die Therapieoptionen.

\footnotetext{
Korrespondenzadresse

Dr. Christiane Reiser

LKH Bregenz, Kinderabteilung

Carl-Pedenz-Straße 2, A-6900 Bregenz

Österreich

E-Mail: Christiane.Reiser@vlkh.net

a+rh 2018; 38: 363-366
}

\section{Fallbericht}

Wir berichten über einen Jungen, bei dem im Alter von acht Jahren die Diagnose eines Hyper-IgE-Syndroms gestellt wurde (DOCK 8-Mutation). Seit Jahren litt der Patient an schwersten kutanen Entzündungen, aggraviert durch schwere Stomatitiden, therapieresistente Candidiasis und chronische Otitiden. Nachdem die jüngere Schwester an der gleichen Erkrankung litt und erfolgreich stammzelltransplantiert worden war, entschied man sich bei unserem Patienten 2013 zur allogenen Stammzelltransplantation. Die immunsuppressive Therapie mit Ciclosporin A konnte im Verlauf nach 18 Monaten beendet werden.

Zu Beginn des Jahres 2016 klagte der Patient über zunehmende Brust- und Kopfschmerzen. In der durchgeführten bildgebenden Diagnostik zeigte sich erstmals das Bild einer Takayasu-Arteriitis ( Abb. 1, CT-Angiografie vier Monate nach Diagnosestellung).

Die ausreichende Kontrolle der vaskulitischen Aktivität gestaltete sich schwierig. Auf die initiale Rituximab-Therapie reagierte der Patient mit einer intensivpflichtigen Intoleranzreaktion. Unter Tocilizumab in Kombination mit Steroiden (Prednisolon in unterschiedlicher Dosierung, max. $1 \mathrm{mg} / \mathrm{kg} / \mathrm{d}$ ) war der Patient zwar klinisch beschwerdefrei, radiologisch zeigte sich allerdings ein Progress der vaskulitischen Aktivität, sodass erneut eine Umstellung erfolgte, nun auf Infliximab (8 mg/kgKG) und MTX, zu Beginn begleitet von Methylprednisolon-Puls-Therapien. Nachdem sich dann in den radiologischen Verlaufskontrollen weiterhin Entzündungsaktivität zeigte ( $\triangleright$ Abb. 2, $\triangleright A b b .3 a)$, wurde das Infliximab-Intervall auf alle vier Wochen ver- kürzt. In der zuletzt durchgeführten Kontrolluntersuchung zeigte sich nun ein milder Rückgang des Wandödems im Aortenbogen ( $\triangle \mathrm{Abb} .3 \mathrm{~b})$.

Als Komplikation entwickelte der Patient nach längerfristiger Steroidtherapie eine arterielle Hypertonie, eine vaskulitisbedingte Nierenarterienstenose konnte ausgeschlossen werden.

\section{Grundlagen zur Takayasu- Arteriitis des Kindesalters (c-TA)}

\section{Krankheitsmonitoring}

Der einzige validierte Score zur Ermittlung der klinischen Krankheitsaktivität ist der PVAS (Pediatric Vasculitis Activity Score), der allerdings Defizite bei den Großgefäßvaskulitiden aufweist (1). Zum laborchemischen Monitoring stellt die Kombination von CRP und BSG immer noch die beste Option dar (2). Es existieren inzwischen noch eine Reihe weiterer neuer Biomarker, die als möglicherweise sinnvolle Parameter zur Messung der Krankheitsaktivität beitragen könnten, so z. B. Matrix-Metalloproteinase (MMP)-9, Interleukin-6 im Serum, und Pentraxin-3 (PTX-3), die aber nur bedingt in der Routinediagnostik verfügbar sind $(3,4)$.

Daher hat weiterhin die radiologische Diagnostik einen sehr hohen Stellenwert, v.a. die MR-Angiografie ist aufgrund der guten Verfügbarkeit und fehlenden Strahlenbelastung in der Pädiatrie als wichtigste Untersuchungsmethode anzusehen. In der Darstellung von Gefäßwandirregularitäten ist die MRA der konventionellen Angiografie überlegen. Aufgrund der hohen Sen- 

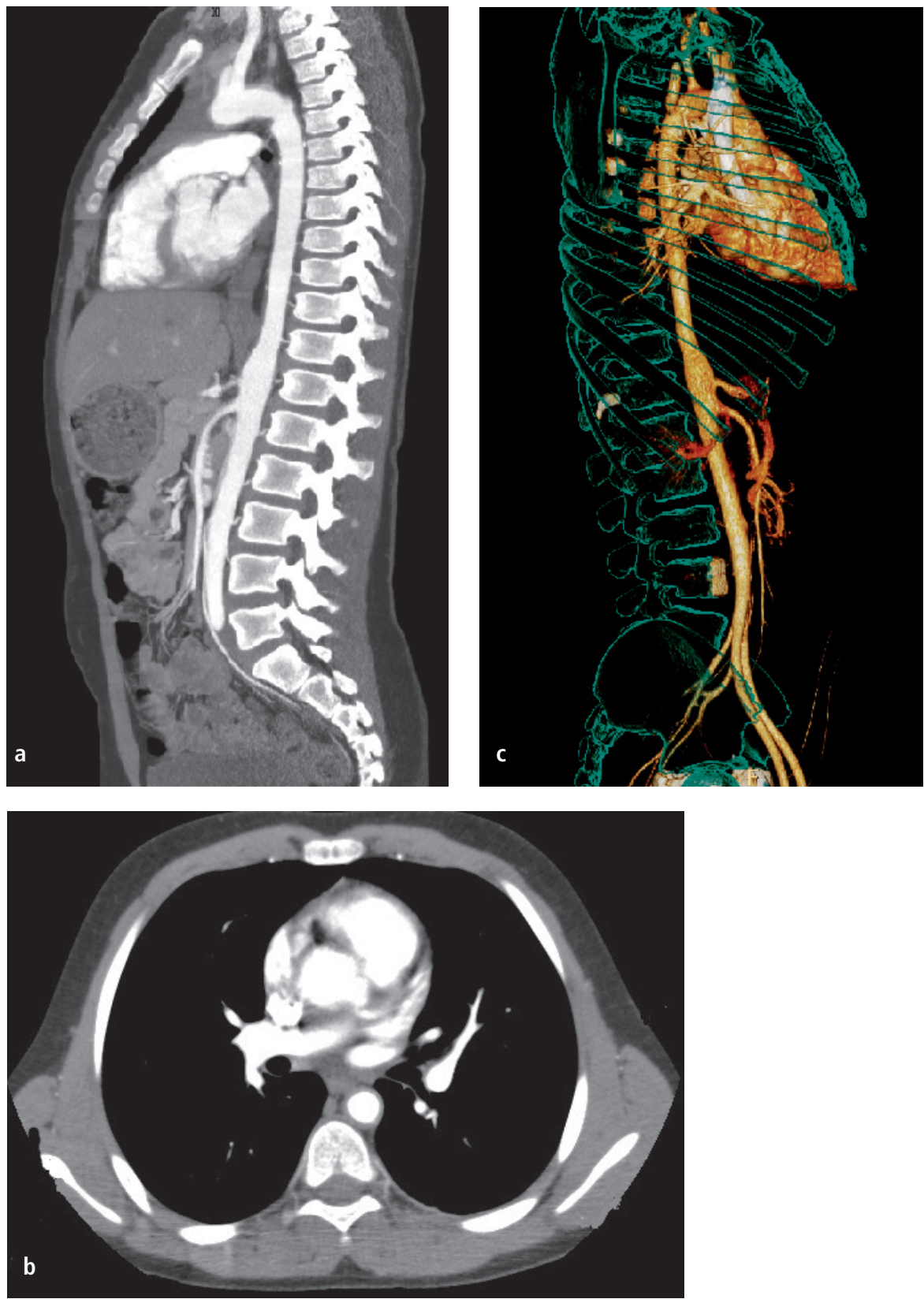

Abb. 1 (a) Sagittale MIP-CT-Angiografie: Wandverdickte Aorta thoracalis und Arteria mesenterica superior. Die Aorta abdominalis ist suprarenal kurzstreckig erweitert; (b) CT-Angiografie axial: zirkulär wandverdickte Aorta thoracalis; (c) CT-Angiografie: fokal erweiterte suprarenale Aorta abdominalis

sitivität der MRA kann allerdings der Grad der Stenose überinterpretiert werden.

Die CT-Angiografie ist in ihrer Aussagekraft der MRA ähnlich, Wandverdickungen und Verkalkungen können typischerweise dargestellt werden. Aufgrund der exzessiven Strahlenbelastung stellt sie im Kindesalter aber nur eine limitierte diagnostische Möglichkeit dar (5).

\section{Hauptkomplikationen}

Die in der Literatur beschriebenen Hauptkomplikationen umfassen die arterielle Hypertonie und die Myokarditis. Klassische neurologische Komplikationen im Kindesalter sind - analog zu unserem Patienten - Kopfschmerzen und Schwindel; bei rund $20 \%$ der betroffenen erwachsenen TA-Patienten tritt ein Apoplex auf (6).
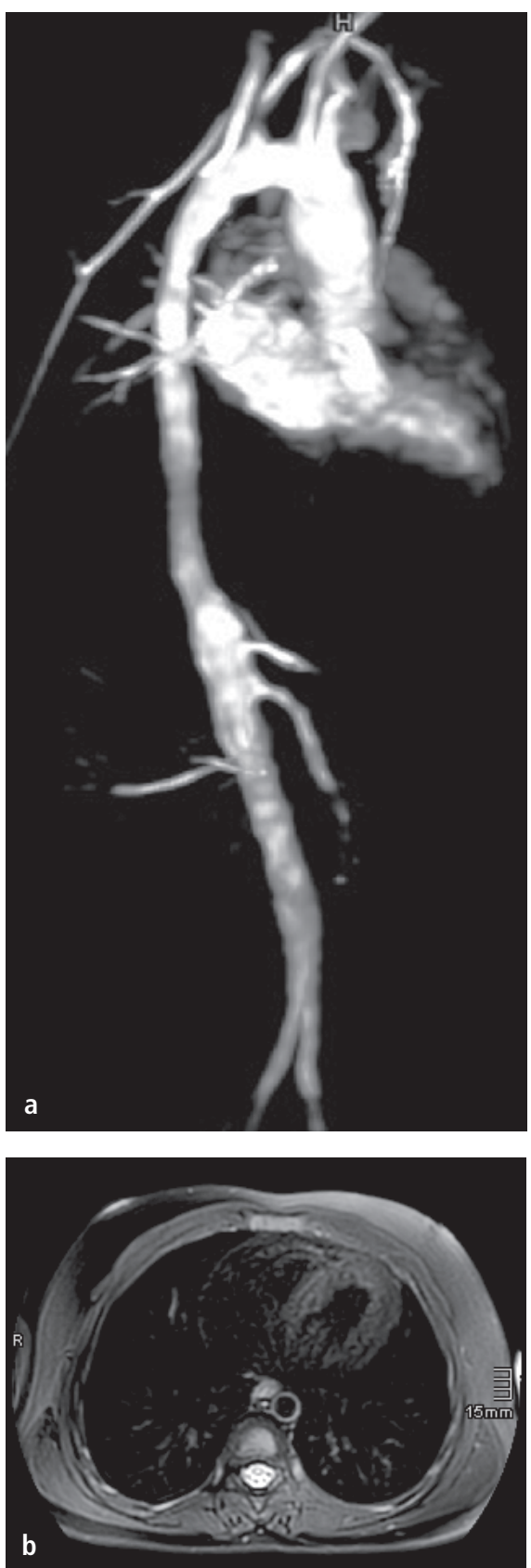

Abb. 2 (a) MR-Angiografie Aorta: kaliberverschmälerte Aorta thoracalis, Kaliberschwankungen der Arteria mesenterica superior, fokal erweiterte suprarenale Aorta abdominalis; (b) MRT, axiale T2-TSE-BLADE mit Fettsättigung: ödematisierte zirkulär verdickte Wand der Aorta thoracalis

\section{Therapieoptionen}

Der frühzeitige Beginn einer immunsuppressiven Therapie ist bei der c-TA unbedingt erforderlich, aber oftmals verzögert aufgrund der unspezifischen Symptome zu Beginn der Erkrankung. 
In der Literatur erwähnte gängige Immunsuppressiva in der Induktionstherapie stellen neben der alleinigen Steroidtherapie die Kombination v.a. mit MTX, Cyclophosphamid oder einem Biologikum dar.

Nach begonnener Steroidtherapie gestaltet sich das Tapering oftmals schwierig. In der Kohorte von Aeschlimann (7) mit 27 Patienten zeigte sich allerdings zum einen, dass auch unter fortgeführter Steroidtherapie regelmäßig Schübe auftreten. Zum anderen konnte hier gezeigt werden, dass unter der Therapie mit Biologika (Infliximab, Adalimumab, Tocilizumab) signifikant seltener Schübe auftraten im Vergleich zur Gruppe der konventionellen Immunsuppressiva. Anderen Berichten zufolge wurden in kleinen Fallserien gute Ergebnisse mit konventionellen Immunsuppressiva, wie Cyclophosphamid zur Induktion und Erhaltungstherapie mit MTX erreicht (8).

Die Stammzelltransplantation stellt eine Alternative dar bei Biologika-resistenten Verläufen von Autoimmunerkrankungen, entsprechende Registerdaten sind verfügbar (ebmt.org). Insbesondere der Graftversus-Autoimmun-Effekt soll bei der allogenen SZT genutzt werden, um eine „Umerziehung" des Immunsystems zu erreichen (9). In der Literatur wird die Option einer autologen Stammzelltransplantation diskutiert (10) und auch erfolgversprechende Daten werden präsentiert.

\section{Verlauf bei unserem Patienten}

In der Zwischenzeit haben wir in den Monozyten des Patienten (Abstrich der Mundschleimhaut) eine NOD2/CARD15-Mutation in Exon 4 nachgewiesen, die bislang noch nicht beschrieben worden ist, während im peripheren Blut des Patienten (100\%iger Spender-Chimärismus) keine Mutation detektierbar war. Mutationen in diesem Gen haben eine besondere Relevanz z.B. beim Morbus Crohn, aber auch beim Blau-Syndrom. „Gain-of-function"-Mutationen führen hier über Veränderungen von NF- $\kappa B$ zu einer vermehrten Produktion von proinflammatorischen $\mathrm{Zy}$ tokinen. Des weiteren wurde beschrieben, dass Polymorphismen in NOD2/CARD15
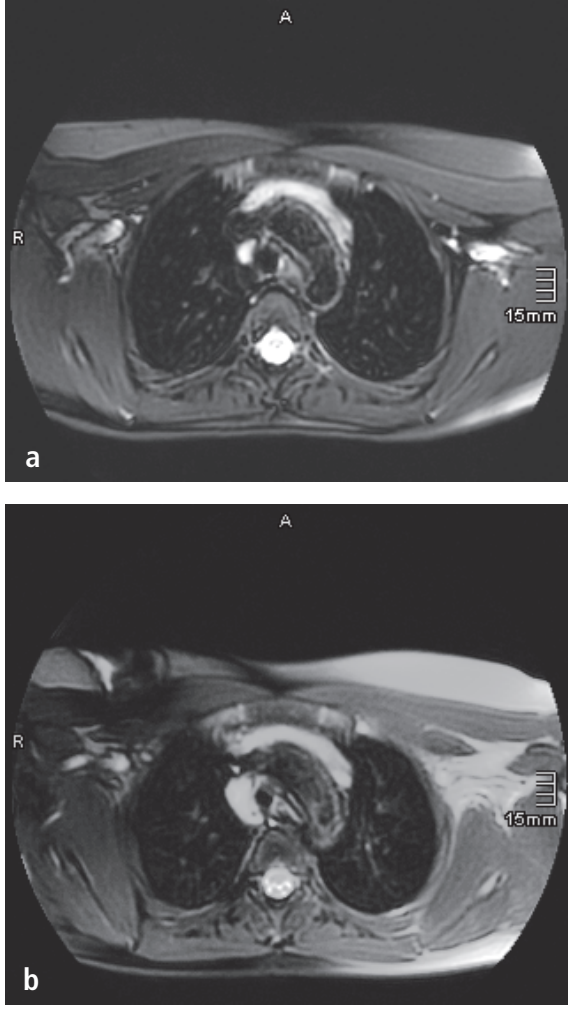

Abb. 3 MR Thorax, axiale T2 BLADE mit Fettsättigung: (a) Untersuchung unter Infliximab $8 \mathrm{mg} /$ kgKG alle acht Wochen; (b) Kontrolluntersuchung nach sechs Monaten nach Verkürzung des Intervalls und Gabe von Infliximab alle vier Wochen: Abnahme des muralen Ödems des Aortenbogens

zu einem erhöhten Auftreten der Graftversus-Host-Disease (GvHD) führen können (11). Bei unserem Patienten liegen nach Transplantation - keine weiteren klinischen Symptome eines M. Crohn oder Blau-Syndroms vor. Beim Blau-Syndrom handelt es sich um eine autoinflammatorische Erkrankung mit der typischen Trias von Arthritis (mit „bulky synovitis"), Uveitis und Dermatitis. In seltenen Fällen kann beim Blau-Syndrom eine Großgefäß-Vaskulitis auftreten (12). Die Arthritis entwickelt sich typischerweise symmetrisch und führt frühzeitig zu Kontrakturen an Zehen und Fingern (Camptodakylie), zusätzlich treten zystische Tenosynovitiden entlang der Hand- und Sprunggelenke auf. Die Uveitis verläuft bei Blau-Patienten häufig schwer, im Verlauf entwickeln viele Patienten eine Sehbehinderung. Bei der Dermatitis werden nicht-verkäsende Granulome histologisch nachgewiesen. Therapeutisch

\section{Zusammenfassung}

Bei unserem Patienten trat nach Stammzelltransplantation bei schwerstem Hyper-lgESyndrom eine Takayasu-Arteriitis auf. Dies stellt unserer Einschätzung nach eine äuBerst seltene Koinzidenz dieser zwei raren immunologischen Erkrankungen dar. Möglicherweise kann die detektierte Mutation im NOD2-Gen hier weitere ätiologische Erkenntnisse, z. B. im Sinne eines Blau-Syndroms, liefern. Prinzipiell herausfordernd bei der Therapie der c-TA ist neben der Auswahl der immunsuppressiven Medikation die Einschätzung der Krankheitsaktivität und die daraus resultierende Frage der Eskalationsnotwendigkeit. Veröffentlichte Fallberichte und kleinere Kohortenstudien zeigen die Schwierigkeit von standardisiertem Vorgehen sowohl hinsichtlich des Monitorings wie auch der Therapieinduktion und der Erhaltungstherapie.

kommen Steroide, Interleukin-1- sowie TNF-alpha-Inhibitoren zum Einsatz.

Weitere Untersuchungen sollen folgen, um die klinische Relevanz dieses Befundes weiter einschätzen zu können und zukünftig gegebenenfalls auch therapeutische Optionen durch eine selektive Zytokinblockade zu eröffnen.

\section{Interessenkonflikt}

Die korrespondieren Autorin gibt an, dass kein Interessenkonflikt besteht.

\section{Einhaltung ethischer Richtlinien}

Die korrespondierende Autorin bestätigt, dass die Familie des Patienten mit der Veröffentlichung der Kasuistik einverstanden ist.

\section{Literatur}

1. Dolezalova et al. Disease activity assessment in childhood vasculitis: development and preliminary validation of the Paediatric Vasculitis Activitiy Score (PVAS). Ann Rheum Dis 2013; 72 (10): 1795-1801.

2. Mathew A, Goel R Kumar S, Danda D. Childhoodonset Takayasu arteritis: an update. Int J Rheum Dis 2016; 19 (2): 116-126. 
3. Dagna L, Salvo F, Tiraboschi M et al. Pentraxin-3 as a marker for disease activity in Takayasu arteritis. Ann Intern Med 2011; 155: 425-433.

4. Sun Y, Ma L, Yan F et al. MMP-9 and IL-6 are potential biomarkers for disease activity in Takayasu's arteritis. Int J Cardiol 2012; 156: 236-238.

5. Papa M, De Cobelli F, Baldissera E et al. Takayasu arteritis: intravascular contrast medium for MR angiography in the evaluation of disease activity. AJR Am J Roentgenol 2012; 198: W279-284.

6. Hoffmann M, Corr P, Robbs J. Cerebrovascular findings in Takayasu disease. J Neuroimaging 2000; 10: 84-90.
7. Aeschlimann $\mathrm{F}$ et al. Childhood Takayasu arteritis: disease course and response to therapy. Arthritis Research \& Therapy 2017; 19: 255

8. Ozen S, Duzova A, Bakkaloglu A et al. Takayasu arteritis in children: preliminary experience with cyclophosphamide induction and corticosteroids followed by methotrexate. J Pediatr 2007; 150: 72-76.

9. Snowden JA, Saccardi R, Allez M et al. Haematopoietic SCT in severe autoimmune diseases: updated guidelines of the European Group for Blood and Marrow Transplantation. Bone Marrow Transplant 2012; 47 (6): 770-790.
10. Daikeler T et al. Haematopoietic stem cell transplantation for vasculitis including Behçet's disease and polychondritis: a retrospective analysis of patients recor. Annals of the Rheumatic Diseases 2007; 66 (2): 202-207.

11. Hyvärinen K, Ritari J, Koskela $S$ et al. Genetic polymorphism related to monocyte-macrophage function is associated with graft-versus-host disease. Scientific Reports 2017; 7: 15666

12. Khubchandani R, Hasija R, Touitou I et al. Blau Arteritis Resembling Takayasu Disease with a Novel NOD2 Mutation. The Journal of Rheumatology 2012; 39 (9): 1888-1892. 